\title{
Resource-saving application of FDTD technique in 3D photonic crystal waveguide calculations
}

\author{
Lavrinenko, Andrei; Tromborg, Bjarne
}

Published in:

Proceedings of 2002 4th International Conference on Transparent Optical Networks

Link to article, DOI:

10.1109/ICTON.2002.1007847

Publication date:

2002

Document Version

Publisher's PDF, also known as Version of record

Link back to DTU Orbit

Citation (APA):

Lavrinenko, A., \& Tromborg, B. (2002). Resource-saving application of FDTD technique in 3D photonic crystal waveguide calculations. In Proceedings of 2002 4th International Conference on Transparent Optical Networks (Vol. 2, pp. 49-52). IEEE. https://doi.org/10.1109/ICTON.2002.1007847

\section{General rights}

Copyright and moral rights for the publications made accessible in the public portal are retained by the authors and/or other copyright owners and it is a condition of accessing publications that users recognise and abide by the legal requirements associated with these rights.

- Users may download and print one copy of any publication from the public portal for the purpose of private study or research.

- You may not further distribute the material or use it for any profit-making activity or commercial gain

- You may freely distribute the URL identifying the publication in the public portal 


\title{
Resource-Saving Application of FDTD Technique in 3D Photonic Crystal Waveguide Calculations
}

\author{
Andrei Lavrinenko, Bjarne Tromborg \\ COM Education and Research Center, Building 345V, DTU, Kgs.Lyngby, DK-2800, Denmark \\ Tel: +4545253811 , Fax: +4545936581 , e-mail: ala@icom.dm.dk
}

\begin{abstract}
This paper presents an algorithm based on the well-known FDTD numerical method which is adapted for 3D problems of transmission and reflection of photonic crystal waveguides, and which effectively saves memory and computing resources. Specific examples showing its validity and effectiveness are presented. Transmission spectra for double 60-degrees bent waveguides reveal narrow resonant transmission in contrast to the broad spectra observed for straight waveguides. Other groups have also registered this feature in recent experiments. The explanation suggested in this paper is that transmission through sharp bends can occur only with pure diffractively guided modes. The dependence of transmission of a coupled-cavity waveguide on the depths of the etched holes is also investigated.
\end{abstract}

Keywords: FDTD technique, photonic crystals, photonic crystal waveguides, integrated circuits.

\section{INTRODUCTION}

The FDTF methods have been widely adopted as very powerful computational techniques in photonic crystal (PC) modeling. They scale linearly either with time or with space, which favor them compared to most other numerical methods for photonic crystai calculations. However, they are very demanding in terms of memory and speed, when they are applied to practical 3D problems such as analysis of transmission spectra of PC waveguides $(\mathrm{PCW})$ in layered structures with $2 \mathrm{D}$ patterning. The methods do not take full advantage of the periodicity, unlike what is done in most band diagram calculations. Use of symmetry conditions may only reduce the memory space by a factor of four for straight PCWs. Other passive system elements, such as Y-splitters, zigzag bends, and vertical couplers have even less symmetry.

The use of FDTD codes to various problems in PCW design has recently been demonstrated in [ $1-10]$. Some of the papers $[1,2,6-8]$ address the modeling of transmission spectra in $3 \mathrm{D}$, which is essential for studying the features of realistic PCWs. This applies in particular to the losses of the PCW modes [5], which do not appear in 2D calculations. A defect mode in the band gap of a 2D photonic crystal propagates without loss.

The transmission loss in 3D PCWs due to the out-of-plane scattering may be simulated in $2 \mathrm{D}$ calculations by making the hole material absorptive. In this way it is possible to obtain transmission spectra in good agreement with experiments [11] and estimate the waveguide loss; but the method cannot be used to predict the transmission losses.

We use the FDTD code ONYX-2 developed by A. Ward and J. Pendry [12] as the background code, and we have modified it for use in $2 \mathrm{D}$ and $3 \mathrm{D}$ PCW problems. A main characteristics of the original code is the coincidence of the space frames for electric and magnetic fields. However, in order to preserve the divergence free leapfrog scheme of the original Lee [13] scheme, the fields are calculated in mesh points being shifted in space by one lattice constant. The detailed description of the procedure and some of our results are presented in Sections 2 and 3.

\section{The FDTD method}

We use first order finite differences approximating the partial derivatives in Maxwell equations. The code applies to media with loss or gain; but here we consider only transparent dielectrics, i.e. the dielectric and magnetic constants are real. In the calculations, the fields are only stored as a function of time for mesh points in detector plates placed directly in a waveguide channel. We avoid allocation of memory for information that is not necessary for a reflection-transmission analysis. The incident field is given by specifying both the electric and the magnetic field [10]. The fields may be designed to excite modes with specific parity.

The adaptation of the ONYX-2 code for PCW problems has required basic modifications of the boundary conditions implemented in the original version [12]. For reflection-free truncating of the computational space we

The work reported in the paper was supported in part by the EU IST project PICCO. 
use the so-called uniaxial perfectly matched layers (PML), based on suggestions by S. Gedney [14]. The advantages of these PMLs over the PMLs introduced by J.P. Berrenger are saving of memory and a more straight procedure for their implementation without splitting of every field component into two parts. The twofold and threefold intersections of the uniaxial absorbing layers have to be treated with special care. The detailed procedure of the PML construction will be given elsewhere.

We have used the modified FDTD code for a variety of different 2D and 3D calculations. Among them are investigations of transmission through straight waveguides of various lengths. The structures are up to 70 lattice constant in length in 2D and 44 lattice constants in 3D. In the vertical direction the PCW consists of 3-4 layers of dielectric material placed on a substrate or suspended in air. The typical size of memory allocated for 2D calculations is about $100 \mathrm{Mb}$ depending on the number of time steps. Usually there were $2^{13}$ steps in time, but the number of time steps was in some cases increased to $2^{14}-2^{15}$ to obtain better resolution. For 3D calculations with reasonable choice of width, thickness and length, the memory requirements arose to $800-900 \mathrm{Mb}$. The work has been done on a HP station with the top processor having $360 \mathrm{MHz}$ working frequency. The standard time for $2 \mathrm{D}$ calculations was up to $12-16$ hours, but for $3 \mathrm{D}$ calculations it was up to 4 days. Some results of the calculations proving the robustness and accuracy of the implemented algorithm are presented in the next Section.

\section{TRANSMISSION SPECTRA FOR PCW}

As the first example we consider transmission through a Si-on-insulator waveguide with two $60^{\circ}$ bends (see Fig. 1); the structure is somewhat similar to the waveguide treated by $\mathrm{E}$ Chow et al in [7]. We use a different normalization in our paper - ratio of transmitted power to input power in the channel. The transmission spectrum shows a sharp peak, which was also found in [7]. This peak occupies only part of the broad transmission band usually observed for a straight waveguide. The broad transmission band is probably due to weakly guided modes such as index guides modes or modes above the light cone. The sharp bends will act as mode strippers, which scatter the weakly guided modes, and the bends will only allow the pure PBG guided modes to be transmitted. These modes have rather flat dispersion curves, and they will therefore give rise to narrow peaks in the transmission spectrum.

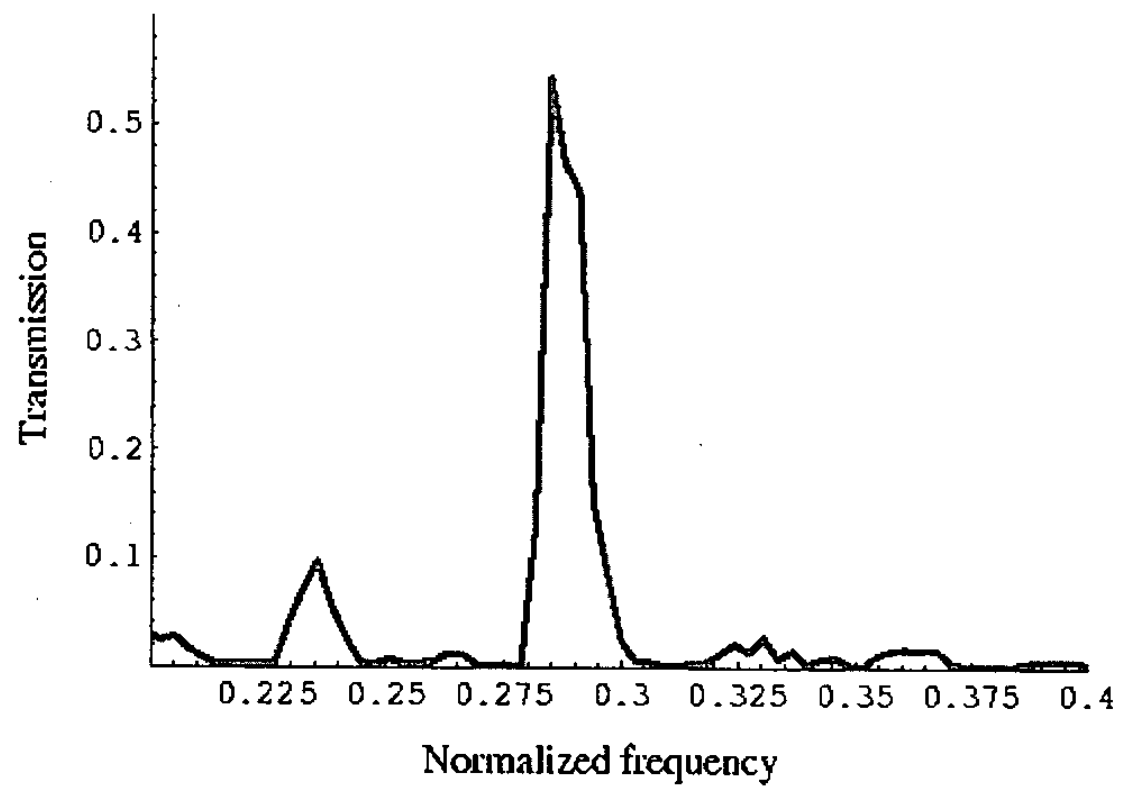

Figure 1. Transmission spectrum of the photonic crystal waveguide with double $60^{\circ}$ bends.

The second example deals with coupled cavity waveguides in a weakly guiding layered system. The prototype of such a system for modeling was taken from [15]. The system comprises a GaAs core in claddings with symmetrical AlGaAs layers mounted on a thick AlGaAs buffer layer. The aim was to see the influence of the depth of the holes penetrating into the substrate on the transmission spectra of the waveguide. The positive role of the substrate for reducing losses was first suggested in [11]. But to the best of our knowledge there have been no explicit calculations of transmission versus depth of the holes. Our results are presented in Fig.2. The overall transmission is rather low due to multiple scattering barriers in the waveguide channel. However, the curves - show a significant improvement of transmission with increasing depth of the holes. For comparison we plot here 
lines for three different depths, $1.4 \mu \mathrm{m}, 1.0 \mu \mathrm{m}$ and $0.8 \mu \mathrm{m}$. Taking into account that the thickness of the cladding-core-cladding sandwich is $0.6 \mu \mathrm{m}$, we obtain direct confirmation of the idea of [11], that the deeper the holes extend into the substrate, the better the transmission. The effect has a threshold and a saturation level. The calculations show very low transmission less than $30 \mathrm{~dB}$ for coupled-cavity PCWs with holes etched only in the guiding sandwich, i.e. with depth $0.6 \mu \mathrm{m}$ in our example. The saturation is reached with holes deeper than $1.2 \mu \mathrm{m}$; the subsequent increase in transmission is practically negligible.

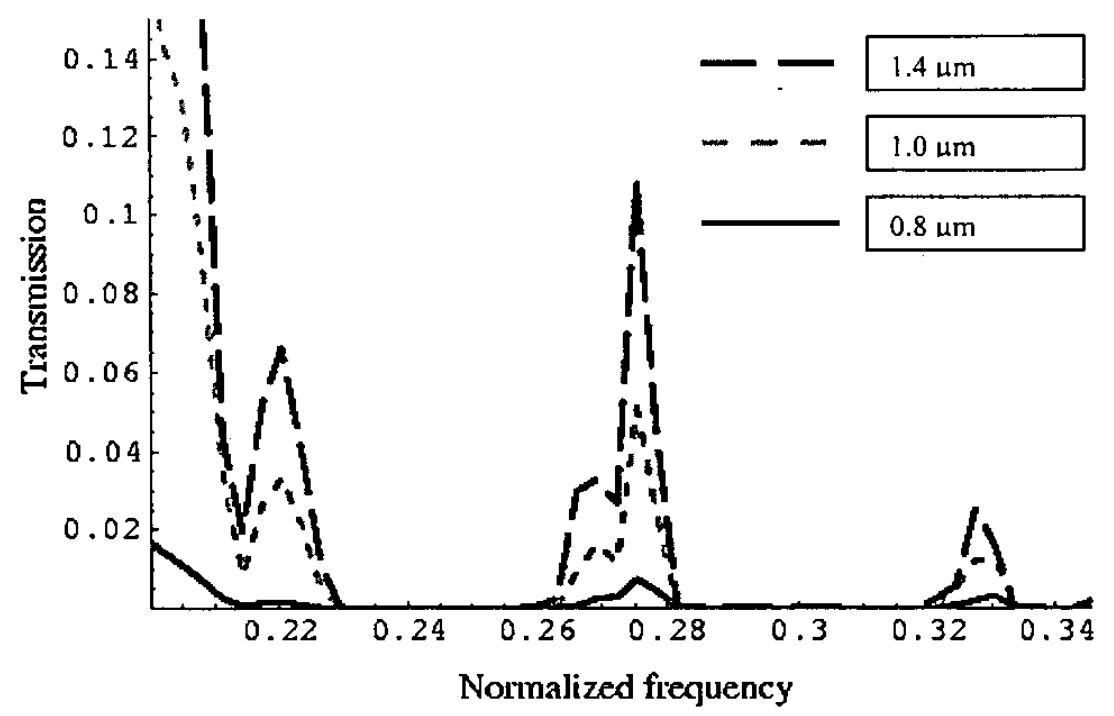

Figure 2. Transmission spectrum of a coupled-cavity waveguide for different depths of the holes.

\section{CONCLUSIONS}

We presented here an effective and memory saving version of the FDTD code ONYX-2 for 3D calculations implemented with special absorbing boundary conditions - uniaxial perfectly matched layers. It allows us to investigate transmission in a broad frequency range for different photonic crystal structures. We found a narrow spectral transmission line for a PCW with double $60^{\circ}$ bends. We argue that the structure only allows transmission of PBG guided mode. These modes have usually a flat dispersion curve and will therefore give rise to a narrow peak in the transmission spectrum. The second example refers to the transmission in coupled-cavity waveguides with weakly guiding structures. The strong dependence of the transmission on the etching depths of the holes defining the PBG structure was confirmed by direct 3D calculations. We estimated the hole depth for which the transmission starts to saturate, as well as the threshold for onset of effective transmission in coupledcavity waveguides.

\section{ACKNOWLEDGEMENTS}

The authors acknowledge stimulating discussions with T. Krauss and fruitful collaboration with T. Sondergaard.

\section{REFERENCES}

[1] S. Fan: Sharp asymmetric line shapes in side-coupled waveguide-cavity systems, Appl. Phys. Lett., vol. 80 , pp. 908-910, Febr. 2002.

[2] Y. Sugimoto, et al.: Fabrication and characterization of different types of two-dimensional AlGaAs photonic crystal slabs, J. Appl. Phys., vol. 91, pp. 922-929, Febr. 2002.

[3] K. Yamada, et al: Improved line-defect structures for photonic-crystal waveguides with high group velocity, Opt. Commun., vol.198, pp. 395-402. Nov. 2001.

[4] M. Loncar, et al:: Methods for controlling positions of guided modes of photonic-crystal waveguides, J. Opt. Soc. Am. B, vol. 9. pp. 1362-1368, Sept. 2001.

[5] M. Agio, C. M. Soukoulis: Ministop bands in single-defect photonic crystal waveguides, Phys. Rev. E, vol.64. pp. 055603-055606, 2001.

[6] T. Ochiai, K. Sakoda: Dispersion relation and optical transmittance of a hexagonal photonic crystal slab, Phys. Rev. B, vol.63, pp. 125107-1251/3, 2001.

[7] E. Chow, et al:: Quantitative analysis of bending efficiency in photonic-crystal waveguide bends at $\lambda=1.55 \mu \mathrm{m}$ wavelengths, Opt. Lett., vol.26, pp. 286-288, March 2001. 
[8] A. Chutinan, S. Noda: Waveguide and waveguide bends in two-dimensional photonic crystal slabs, Phys. Rev. B. vol.62, pp. 4488-4492, Aug. 2000.

[9] A. Adibi, et al.: properties of the slab modes in photonic crystal optical waveguides, J. Lightwave Technol., vol. 18. pp.1554-1564, Nov. 2000.

[10] M. Qiu, S. He: Numerical method for computing defect modes in two+dimensional photonic crystals with dielectric or metallic inclusions, Phys. Rev. B, vol.61, pp. 12871-12876, May 2000.

[11] H. Benisty, et al.: Radiation losses of waveguide-based two-dimensional photonic crystals: Positive role of the substrate, Appl. Phys. Lett., vol. 76. pp. 532-534, Jan. 2000.

[12] A.J. Ward, J.B. Pendry, A program for calculating photonic band structures, Green's functions and transmission/reflection coefficients using a non-orthogonal FDTD method, Comp. Phys.Comm., vol. 128, pp. $590-621,2000$.

[13] A. Taflove: Computational Electrodynamics: The Finite-Difference Time-Domain Method, Boston, London: Artech House, 1995.

[14] S. D. Gedney: An anisotropic perfectly matched layer - absorbing medium for the truncation of FDTD lattices, IEEE Trans. Ant. Prop., vol.44, pp.1630-1639, Dec. 1996.

[15] S. Olivier, et al.: Miniband transmission in a photonic-crystal coupled-resonator optical waveguide, Opt. Lett., vol. 26, pp. 1019-1021, 2001. 